\title{
Advanced systolic heart failure and Mycobacterium avium complex (MAC) infection: Proposed immunologic mechanism, diagnostic dilemma and treatment considerations
}

\author{
Leon Varjabedian ${ }^{1}$, Shehzad Merchant ${ }^{2}$, Melissa Danek ${ }^{3}$ \\ 1. Department of Cardiovascular diseases, University at Buffalo, NY, USA. 2. Infectious Diseases Division, Department of \\ Medicine, Buffalo General Medical Center, University at Buffalo, NY, USA. 3. Department of Pharmacy, Kaleida \\ Health-Buffalo General Medical Center, Buffalo, NY, USA
}

Correspondence: Clinical Assistant Professor, Shehzad Merchant. Address: Infectious Diseases Division, Department of Medicine, Buffalo General Medical Center, University at Buffalo, 100 High Street, B-8, Buffalo, NY 14203, USA. Email: sm289@buffalo.edu

Received: June 26, 2015

Accepted: August 12, 2015

Online Published: August 18, 2015

DOI : $10.5430 /$ crim.v2n3p89

URL: http://dx.doi.org/10.5430/crim.v2n3p89

\begin{abstract}
Mycobacterium avium complex (MAC) infection is well recognized in patients with cellular immunodeficiency. Recognition of its morbidity in non-immunocompromised patients has emerged in the last few decades. Underlying chronic pulmonary disease, bronchiectasis, cigarette smoking, cystic fibrosis and chest wall deformities are well-recognized predisposing conditions. Weight loss, dyspnea, cough, fatigue and sputum production are the most common presenting symptoms. We present a case of culture proven MAC infection in an elderly female whose dyspnea and fatigue for a considerable period was inappropriately attributed to chronic heart failure exacerbations necessitating frequent hospital admissions. Despite optimal medical management, clinical improvement was not achieved until a broncho-alveolar lavage (BAL) culture grew MAC and antibiotic therapy targeted against MAC was introduced. Our case report proposes chronic systolic heart failure as a new (not well-recognized) predisposing condition for MAC infection in elderly patients. Because clinical diagnosis can be delayed for weeks, we recommend obtaining sputum/BAL cultures if no clinical improvement is achieved in heart failure patients despite optimal management. High Resolution Computerized Tomography (HRCT) of the chest may also provide meaningful information. We also emphasize the importance of adjusting antibiotics, especially clarithromycin (CLR), based on renal function and tailoring antibiotic management according to serum drug levels to avoid potential liver toxicity associated with CLR use.
\end{abstract}

\section{Keywords}

Mycobacterium avium complex, Congestive heart failure, Pro-inflammatory cytokines, Clarithromycin, Therapeutic drug levels, Cholestatic hepatitis

\section{I ntroduction}

Disseminated disease is the typical presentation of Mycobacterium Avium Complex (MAC) in human immunodeficiency virus (HIV) related immunodeficient patients. However, abundant literature has surfaced in the last two decades 
recognizing MAC as a significant cause of pulmonary disease in patients with no underlying immunosuppressive disorders. Despite recognition of certain risk factors, the pathophysiology behind a small patient group (relative to the ubiquity of exposure to MAC) presenting with clinically significant disease is not well known. Defects in the local defense mechanism of the lungs are a proposed mechanism.

In immunocompetent subjects, Chitty et al. have suggested five categories based on the different clinical and radiologic patterns associated with pulmonary disease caused by MAC ${ }^{[1]}$. These categories include Type 1) asymptomatic colonization, Type 2) simple disease with symptoms and minimal, limited radiographic changes that includes Lady Windermere syndrome, Type 3) complex progressive disease with symptomatic and radiographic deterioration with an underlying cavitary disease $\{3 \mathrm{~A}\}$ or an underlying nodular bronchiectasis $\{3 \mathrm{~B}\}$, Type 4 ) Complicated disease, with failure of initial therapy or multiple recurrences and Type 5) MAC Hypersensitivity Pneumonitis (Hot Tub Lung). Distorted lung architecture secondary to bullous emphysema, stage III-IV sarcoidosis, bronchiectasis and pneumoconiosis can predispose normal subjects to MAC superinfection (types 3 and 4 disease) ${ }^{[2,3]}$. Complex progressive disease with symptomatic and radiographic deterioration, is the most common presentation that occurs in middle-aged, or older men with underlying chronic obstructive pulmonary disease (COPD), alcoholism, and lung cancer, or who have undergone gastrectomy (types

3 and 4 disease) ${ }^{[4,5]}$. The nodular bronchiectatic variety has predilection in elderly nonsmoking white women ${ }^{[6]}$ with lingular or right middle lobe involvement (types 2 and 3B). This presentation was also defined in patients having undergone coronary artery bypass surgery (type 3B) ${ }^{[7]}$.

However, congestive heart failure (CHF) is not recognized as a predisposing factor for acquiring pulmonary MAC infection. We present a patient whose respiratory symptoms were long attributed to underlying advanced systolic heart failure, until reversed by an antibiotic regimen aimed against MAC. We also discuss the potential utility of monitoring clarithromycin (CLR) drug levels in patients who are being treated for pulmonary MAC disease.

\section{Case report}

A 77-year-old female with chronic kidney disease, non-ischemic dilated cardiomyopathy, New York Heart Association Class III-IV (NYHA III-IV) with an ejection fraction (EF) of 25\%, on optimal medical therapy and status post defibrillator (AICD) placement, presented with a 6-month history of cough, intermittently productive of white/yellow phlegm and worsening shortness of breath. She had no fever, night sweats or chills. These symptoms were first attributed solely to CHF, which lead to recurrent hospitalizations (in the last six months) and only a partial response (to medical therapy directed towards CHF) was noted. Due to worsening cough, fatigue and yellow phlegm, a prolonged course of levofloxacin for "chronic bronchitis" was futile although a brief course of azithromycin prior to this admission was "somewhat beneficial”. Physical exam was notable for rales at both lung bases and 92\% oxygen saturation on room air. Laboratory results showed a normal white count and differential. Chest X-ray showed increased interstitial markings (see Figure 1). High-resolution computerized tomography (HRCT) of the chest without contrast showed bilateral centrilobular pulmonary nodules with tree in bud appearance (see Figure 2) and bilateral lower lobe mural bronchial thickening (see Figure 3) with mucous plugging (see Figure 4). The Infectious Diseases service was consulted and they suggested sending sputum and broncho-alveolar lavage for mycobacterial culture and microscopic examination for acid fast bacilli (AFB). Specimens were smear positive for AFB and cultures grew MAC. Routine bacterial and fungal cultures were without growth. A diagnosis of Lady Windermere syndrome was made, and the patient was started on daily dosing of CLR, ethambutol and rifabutin. During follow up visits, the patient noticed a significant improvement in cough and sputum production and a moderate improvement in dyspnea (NYHA I-II). 


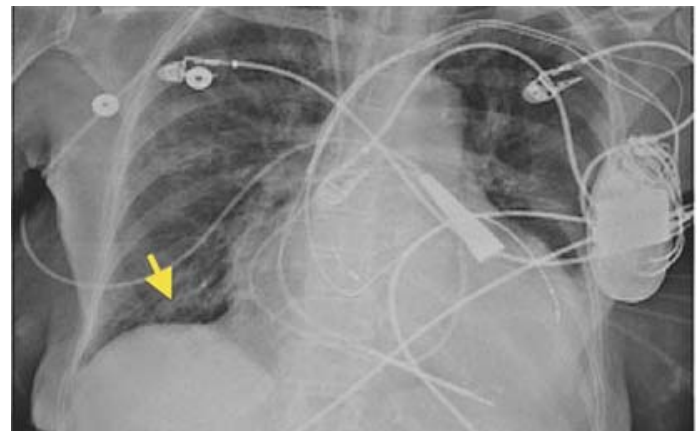

Figure 1. Right lower lobe infiltrate and increased interstitial markings

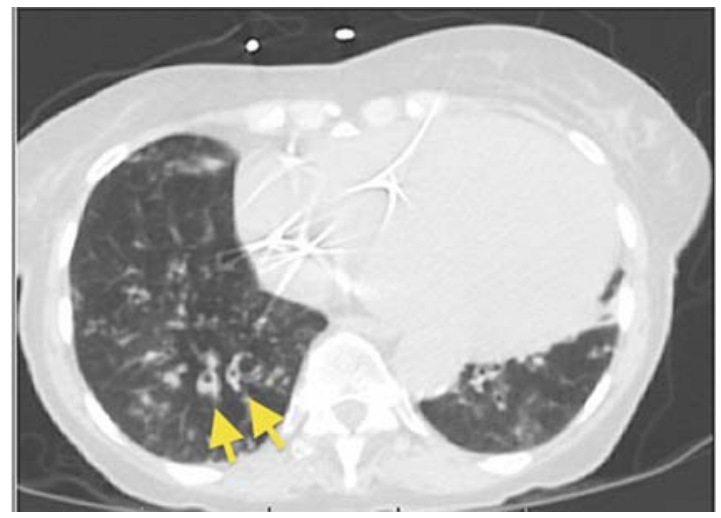

Figure 3. Bronchiectatic changes

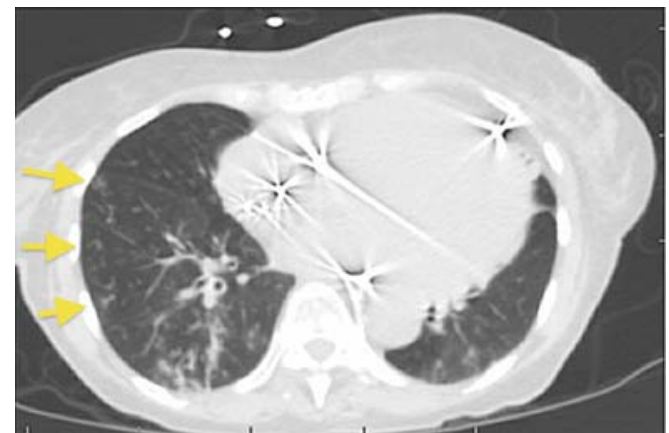

Figure 2. Tree in bud appearance on CT thorax without contrast

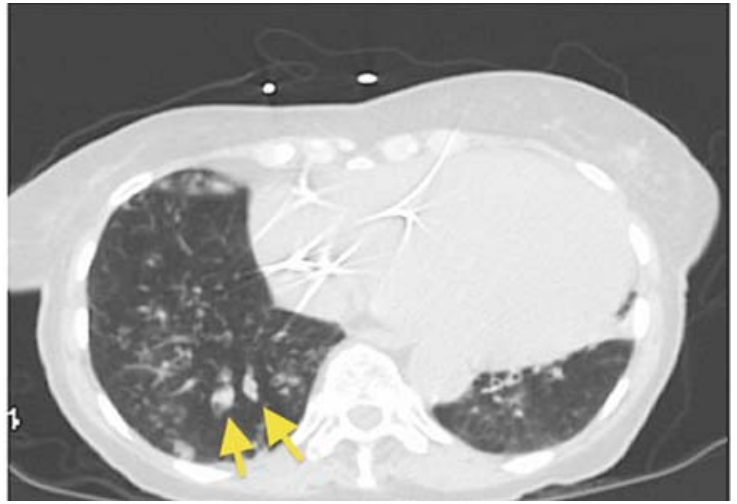

Figure 4. Mucous plugging

Two months into therapy, she was admitted for cholestatic hepatitis (Total bilirubin $5.1 \mathrm{mg} / \mathrm{dl}$, direct bilirubin $3.1 \mathrm{mg} / \mathrm{dl}$, alkaline phosphatase 152 unit/L, AST 175 unit/L, ALT 160 unit/L) with an acute onset of nausea, vomiting, and metallic taste. A few weeks prior to emergence of these symptoms, rifabutin therapy had been stopped as there was a concern for uveitis. Also, to optimize her CHF, her diuretics were constantly being adjusted prior to this admission and on admission, creatinine had worsened (from $1.62 \mathrm{mg} / \mathrm{dl}$, creatinine clearance $\{\mathrm{CrCL}\}$ of $35 \mathrm{ml} / \mathrm{min}$ during last admission, to $2.7 \mathrm{mg} / \mathrm{dl}$, CrCL of $17 \mathrm{ml} / \mathrm{min}$ on current admission). She had a normal right upper quadrant ultrasound. Viral, immunologic, and other hepatitis etiologies were deemed unlikely based on negative serologies, which included hepatitis B \{HBsAg, anti-HBc\}, hepatitis $\mathrm{C}$ \{anti-HCV\}, cytomegalovirus $\{\mathrm{CMV}\}$ and Ebstein Barr virus $\{\mathrm{EBV}\}$, anti-nuclear antibody $\{$ ANA , anti-smooth muscle antibody $\{$ ASMA\}, and liver kidney microsomal Type-1 \{LKM-1\} antibodies. Clarithromycin induced cholestatic hepatitis was suspected when measured serum CLR levels were increased 3-fold (random level $10.6 \mu \mathrm{g} / \mathrm{ml}$, suggested peak range 3.0-4.0 $\mu \mathrm{g} / \mathrm{ml}$ ). Liver function tests returned to baseline after the CLR dose was adjusted for creatinine clearance with no further gastrointestinal symptoms, thereby substantiating this consideration and patient's respiratory status continued to improve on the combination of CLR and ethambutol.

\section{Discussion}

The clinical presentation of MAC in immunocompetent adults can be broadly divided into two major clinical categories based on the presence of underlying lung disease or absence of antecedent pulmonic pathology or smoking history ${ }^{[8]}$. These can be divided into 5 clinical subcategories based on microbiologic criteria and dichotomous radiologic findings ${ }^{[1]}$. Our patient was diagnosed with pulmonary MAC infection based on the American Thoracic Society (ATS) and IDSA (Infectious Disease Society of America) Statement ${ }^{[9]}$. She would be classified as complex progressive disease with symptomatic and radiographic deterioration (type 3B) based on the classification proposed by Chitty et al. ${ }^{[1]}$. Our patient did not have the skeletal abnormalities such as scoliosis or pectus excavatum as a defined risk factor ${ }^{[16,17]}$ predisposing to 
this condition (Type 3B). She did not have distorted lung architecture due to stage III/IV sarcoidosis, pneumoconiosis, bronchiectasis, bullous emphysema, cystic fibrosis or other conditions known to predispose patients towards MAC superinfection, usually Types 3 and $4{ }^{[2,3]}$. She was not known to be alcoholic, smoker or have obstructive lung disease as these conditions give rise to cavitary (type 3A) disease.

In our patient, lower lobes were predominantly involved, which was thought to be related to heart failure exacerbations on her prior admissions. Historically, it appears that the middle lobe and lingula are more predisposed to MAC pulmonary disease because of their particular anatomic structures and absence of collateral ventilation ${ }^{[10]}$.

End stage renal disease (ESRD) impairs different aspects of cell mediated immune response, the combination of uremic toxins and malnutrition in ESRD can ameliorate host defense mechanisms against intracellular organisms such as MAC by affecting lymphocyte and granulocyte function regardless of dialysis status. In fact, there are reported cases of disseminated and localized MAC infection in patients (without HIV) after starting peritoneal dialysis. Our patient had chronic kidney disease (CKD) and was not on dialysis yet. Chronic renal failure has been identified as a possible risk factor for NTM disease in retrospective cohort studies and literature reviews ${ }^{[11-13]}$ and therefore, it is possible that CKD was a predisposing factor as well towards a MAC infection.

Association between systolic heart failure and MAC infection has not been previously reported. We speculate that advanced congestive heart failure may be a predisposing condition for MAC in non-immunocompromised elderly thin patients. Interstitial edema in the lower lobes may predispose to decreased ventilation, creating a nidus for infection. Decreased aeration may lead to a collection of pro-inflammatory cytokines. Our hypothesis stems from data published by Yamazaki et al. ${ }^{[14]}$ who showed that patients with MAC infection with neither predisposing lung condition nor immunodeficiency, had pulmonary function test (PFT) findings that suggested presence of air trapping and disproportionate ventilation. This may be a reflection of the intensity of neutrophil-related inflammation of the small airways in this disorder. They also showed ${ }^{[20]}$ that BAL fluid in patients with MAC disease had increased neutrophils, activated CD4 lymphocyte cell counts, and pro-inflammatory cytokines including Tumor necrosis factor (TNF), Interleukins (IL-1, IL-6, and IL-8) and neutrophil elastase (NE) concentration ${ }^{[15]}$. These same inflammatory markers, specially TNF-a and IL-6, are elevated in advanced heart failure and have causative etiology in myocyte apoptosis, left ventricular dysfunction and remodeling ${ }^{[16]}$. We hypothesize that the same milieu that actively plays part in heart failure progression might also predispose to pulmonic MAC infection.

Heart failure (HF) has a complex pathophysiology. Besides neuro-hormonal and autonomic changes, it is proposed that $\mathrm{CD}^{+}$lymphocytes play a role in extracellular matrix remodeling; therefore HF is recognized as a chronic low grade inflammatory state ${ }^{[17]}$. Several pro-inflammatory cytokines are increased in heart failure ${ }^{[15,16]}$. IL-8 correlates with adverse prognostic outcomes in heart failure patients ${ }^{[20]}$. IL-8 is induced in the myocardium during myocardial injury ${ }^{[24]}$. In addition to its role in promoting inflammatory cascades that may lead to a worsening of heart failure ${ }^{[22]}$, high IL-8 levels may predispose to decreased host defense mechanisms at the level of the alveoli, hence leading to increased susceptibility towards opportunistic infections. It is not known if recurrent interstitial edema that is seen in heart failure exacerbations can alter the local immune defense mechanism at the level of the alveoli and thus predispose to MAC infection. It is however reported that certain lung conditions like pulmonary alveolar proteinosis (PAP) predispose to MAC infection by impaired $\mathrm{T}$ cell activation related to reduce granulocyte macrophage colony-stimulating factor (GM-CSF) function ${ }^{[23]}$. The localized decrease in defense mechanisms is even proposed in slender old women who lack estrogen as ovariectomized mice were more susceptible to MAC infection ${ }^{[24]}$. The mechanism is believed to be related to decreased macrophage proliferation. There is clearly more that must be learned about this possible association between CHF and MAC lung disease. Our case can establish grounds for future molecular or physiologic trials to try and define a true pathophysiologic predisposition towards MAC infection in advanced heart failure patients. 
The ATS/IDSA statement recommends triple antibiotic therapy, (CLR, ethambutol and a rifamycin) for nodular or bronchiectatic MAC pulmonary disease ${ }^{[25]}$. It is generally recommended that the CLR dose be adjusted for patients with decreased creatinine clearance $(\mathrm{CrCl}<30 \mathrm{ml} / \mathrm{min})^{[26]}$ and in retrospect, perhaps our patient should have been started on a lower dose of CLR, based on her age and already known chronic kidney disease. At that point in time, her $\mathrm{CrCl}$ hovered between 30-40 $\mathrm{ml} / \mathrm{min}$ and with concern for achieving insufficient drug concentrations; she was discharged on the standard dose of $500 \mathrm{mg}$ twice a day. In one study, attempts at increasing CLR doses (to 1,000 mg twice a day), in hopes of achieving a better clinical response, was difficult to tolerate, in a group of elderly patients with low body mass ${ }^{\text {[27] }}$. It is well reported that adherence to guideline driven therapy in Pulmonary non tuberculous mycobacterium (PNTM), especially to duration of macrolide use, is poor ${ }^{[28]}$. CLR, a macrolide with broad applications due to its activity against bacteria causing pneumonia, is usually well tolerated. However, cholestatic liver injuries have been reported ${ }^{[27,29-31]}$, and fulminant liver failure necessitating liver transplant has also been described ${ }^{[32,33]}$. Some of these cases occurred after a short period of drug exposure. In vitro studies suggest that the formation of toxic metabolites by cytochrome P450 followed by local depletion of glutathione may be a cause for hepatocellular injury due to macrolide antibiotics ${ }^{[34]}$. In our case, CLR treatment was indicated for a longer period of time. In cases of suspected drug toxicity, it is difficult to identify the causative agent, especially when patients are receiving multiple drugs with possible interactions and similar side effect profiles. In this case, we identified a high CLR serum level, in the absence of other hepatotoxic drugs since rifabutin was stopped a few weeks prior to admission. This, in combination with the resolution of symptoms and normalization of liver function tests with an adjustment in the CLR dose, strongly supports CLR as being responsible. High CLR levels were also reported in the study by Wallace et al. when higher doses of CLR were attempted and poorly tolerated ${ }^{[27]}$.

The use of currently recommended regimens and doses in MAC lung disease leads to important pharmacologic interactions that may result in low drug concentrations and area under the curve (AUC) especially for macrolides ${ }^{[35]}$. The necessity for monitoring macrolide levels to minimize toxicity and maximize efficacy is debatable. CLR drug levels are not typically monitored at our institution (as in many academic centers), necessitating measurement at a specialty laboratory. There is conflicting data on the clinical usefulness of therapeutic drug monitoring (TDM), in the treatment of lung disease due to nontuberculous mycobacteria (NTM). After oral administration, CLR is highly concentrated in the alveolar macrophages via transport across the epithelial lining from the blood. The mean tissue concentrations are 2 to 20 times greater than the plasma concentrations, and the alveolar macrophage concentrations are 400 times greater than the plasma concentrations for CLR ${ }^{[36]}$. In one study that measured CLR minimum inhibitory concentration (MIC) values, and plasma peak CLR concentration/MIC ratios, the authors did not see a difference in drug levels between patients who showed a favorable microbiological response and those who showed an unfavorable microbiological response ${ }^{[37]}$, suggesting TDM may not be beneficial in the therapeutic management of patients with MAC lung disease ${ }^{[37]}$. Other authors critique problems with methods and conclusions with TDM for MAC infections ${ }^{[38]}$. Taking into account the pharmacokinetics of macrolides, microbial kill by these agents is linked to the area under the curve (AUC)-to-MIC ratio ${ }^{[39,40]}$. In a retrospective treatment study of pulmonary MAC infection, Kobashi et al. ${ }^{[41]}$ demonstrated clinical and microbiological (including sputum conversion rate) improvement with high dose CLR compared to low dose CLR, despite MAC MICs to CLR being similar. However, higher doses of CLR were also associated with higher incidence of gastrointestinal adverse reactions without the need to discontinue CLR administration.

Alternative dosing strategies have been recommended for this triple drug regimen for the treatment of MAC. Intermittent dosing (CLR 1,000 mg, ethambutol $25 \mathrm{mg} / \mathrm{kg}$ and rifabutin 150-300 $\mathrm{mg}$ all three times a week) was evaluated in non-HIV patients with MAC lung disease. Thirty-two of the 41 patients who completed 6 months of therapy had conversion of their sputum to negative during this time period. Significant adverse effects were still problematic and required further dose adjustments in these patients as well ${ }^{[42]}$. A more recent retrospective review in patients with nodular/bronchiectatic MAC lung disease demonstrated the 3 times per week drug regimen to be significantly better tolerated and as efficacious as a daily regimen (though statistical comparison was not done) ${ }^{[43]}$. Furthermore, the ATS/IDSA statement actually recommends this 3 times per week regimen as initial therapy in patients with nodular/bronchiectatic disease ${ }^{[25]}$. 
Therefore, based on the literature and the case in this report, some important points should be considered with regards to drug dosing and monitoring. When treating an elderly patient with borderline renal function, it is extremely important to consider the dose of CLR being used. Intermittent dosing may be a preferable strategy in these patients as it allows for overall less drug exposure and seems to be better tolerated. Close monitoring of renal and hepatic function is absolutely necessary, especially if the patient is on other drugs, which may affect either of these systems. It seems reasonable to consider checking a CLR level in situations when drug toxicity is suspected. Consideration may be given to routinely following CLR levels to maximize the chances of avoiding toxicity and thereby enabling the continued use of CLR, a critical drug for affecting a cure in MAC infection.

\section{Conclusion}

Pulmonary MAC is emerging to cause serious morbidity in immunocompetent individuals; the rise of incidence in industrialized countries in patients with no predisposing factors is not fully understood. Despite the vast number of predisposing conditions reported in the literature, our case is the first case to suggest congestive heart failure to be more than an incidental co-morbid condition. Even though our paper does not allow us to draw a pathophysiologic relationship between CHF and MAC infection, we believe that chronic systolic heart failure may be an unrecognized predisposing condition for pulmonary MAC disease. Our case raises the importance of considering MAC in chronic heart failure patients in whom worsening of respiratory symptoms cannot be easily attributed to decompensated heart failure. Once reversible etiologies of heart failure, such as ischemia, valvular heart disease and arrhythmias are addressed or ruled out, a diagnostic protocol should be initiated, to look at non-cardiac (and particularly infectious) causes of cardiac-respiratory failure whereby, the possibility of underlying MAC infection should particularly be considered. In these patients, we propose sending sputum cultures or preferably a BAL (bronchoalveolar lavage) looking for MAC infection. It is also essential to declare failure of guideline directed medical therapy before ordering any imaging work up for MAC. In the appropriate setting and in the likely predisposed population, a high resolution CT (HRCT) chest, along with sputum/BAL with appropriate microbiologic diagnostics may help identify an underlying mycobacterial infection.

This case also demonstrates the importance of closely following renal function as well as liver function tests during long-term CLR treatment and the utility of checking CLR blood levels to prevent or diagnose drug induced cholestatic hepatitis, especially with decreased or fluctuating renal function.

\section{Acknowledgments}

We thank Dr. Thomas A. Russo, Professor and Chief of Infectious Disease at University at Buffalo for reviewing the manuscript and his kind support.

\section{Competing interests}

The authors declare that they have no competing interests.

\section{References}

[1] Chitty SA, Ali J. Mycobacterium avium complex pulmonary disease in immunocompetent patients. South Med J. 2005; 98(6): 646-52. PMid:16004172 http://dx.doi.org/10.1097/01.SMJ.0000154311.60057.56

[2] Oliver A, Maiz L, Canton R, et al. Nontuberculous mycobacteria in patients with cystic fibrosis. Clin Infect Dis. 2001; 32(9): 1298-303. PMid:11303264 http://dx.doi.org/10.1086/319987

[3] Marras TK, Daley CL. Epidemiology of human pulmonary infection with nontuberculous mycobacteria. Clin Chest Med. 2002; 23(3): 553-67. http://dx.doi.org/10.1016/S0272-5231(02)00019-9

[4] Rosenzweig DY. Pulmonary mycobacterial infections due to Mycobacterium intracellulare-avium complex. Clinical features and 
course in 100 consecutive cases. Chest. 1979; 75(2): 115-9. PMid:421545 http://dx.doi.org/10.1378/chest.75.2.115

[5] Wolinsky E. Nontuberculous mycobacteria and associated diseases. Am Rev Respir Dis. 1979; 119(1): 107-59. PMid:369415

[6] Wittram C, Weisbrod GL. Mycobacterium avium complex lung disease in immunocompetent patients: radiography-CT correlation. Br J Radiol. 2002; 75(892): 340-4. PMid:12000692 http://dx.doi.org/10.1259/bjr.75.892.750340

[7] Tryfon S, Angelis N, Klein L, et al. Lady Windermere syndrome after cardiac surgery procedure: a case of Mycobacterium avium complex pneumonia. Ann Thorac Surg. 2010; 89(4): 1296-9. PMid:20338366 http://dx.doi.org/10.1016/j.athoracsur.2009.08.066

[8] Plotinsky RN, Talbot EA, von Reyn CF. Proposed definitions for epidemiologic and clinical studies of Mycobacterium avium complex pulmonary disease. PLoS One. 2013; 8(11): e77385. PMid:24265675 http://dx.doi.org/10.1371/journal.pone.0077385

[9] Diagnosis and treatment of disease caused by nontuberculous mycobacteria. This official statement of the American Thoracic Society was approved by the Board of Directors, March 1997. Medical Section of the American Lung Association. Am J Respir Crit Care Med. 1997; 156(2 Pt 2): S1-25. PMid:9279284

[10] Dhillon SS, Watanakunakorn C. Lady Windermere syndrome: middle lobe bronchiectasis and Mycobacterium avium complex infection due to voluntary cough suppression. Clin Infect Dis. 2000; 30(3): 572-5. PMid:10722445 http://dx.doi.org/10.1086/313726

[11] Ergin A, Hascelik G. Non tuberculous mycobacteria (NTM) in patients with underlying diseases: results obtained by using polymerase chain reaction restriction enzyme analysis between 1997-2002. New Microbiol. 2004; 27: 49-53. PMid:14964405

[12] Ingram CW, Tanner DC, Durack DT, et al. Disseminated infection with rapidly growing mycobacteria. Clin Infect Dis. 1993; 16: 463-471. PMid:8513048 http://dx.doi.org/10.1093/clind/16.4.463

[13] Wallace RJ Jr, Brown BA, Onyi GO. Skin, soft tissue, and bone infections due to Mycobacterium chelonae chelonae: importance of prior corticosteroid therapy, frequency of disseminated infections, and resistance to oral antimicrobials other than clarithromycin. J Infect Dis. 1992; 166: 405-412. http://dx.doi.org/10.1093/infdis/166.2.405

[14] Yamazaki Y, Kubo K, Fujimoto K, et al. Pulmonary function tests of Mycobacterium avium-intracellulare infection: correlation with bronchoalveolar lavage fluid findings. Respiration. 2000; 67(1): 46-51. PMid:10705262 http://dx.doi.org/10.1159/000029462

[15] Yamazaki Y, Kubo K, Sekiguchi M, et al. Analysis of BAL fluid in M. avium-intracellulare infection in individuals without predisposing lung disease. Eur Respir J. 1998; 11(6): 1227-31. PMid:9657559 http://dx.doi.org/10.1183/09031936.98.11061227

[16] von Haehling S, Schefold JC, Lainscak M, et al. Inflammatory biomarkers in heart failure revisited: much more than innocent bystanders. Heart Fail Clin. 2009; 5(4): 549-60. PMid:19631179 http://dx.doi.org/10.1016/j.hfc.2009.04.001

[17] Mann DL. Inflammatory mediators and the failing heart: past, present, and the foreseeable future. Circ Res. 2002; 91(11): 988-98. PMid:12456484 http://dx.doi.org/10.1161/01.RES.0000043825.01705.1B

[18] Yndestad A, Damas JK, Oie E, et al. Systemic inflammation in heart failure-the whys and wherefores. Heart Fail Rev. 2006; 11(1): 83-92. PMid:16819581 http://dx.doi.org/10.1007/s10741-006-9196-2

[19] Yndestad A, Damas JK, Oie E, et al. Role of inflammation in the progression of heart failure. Curr Cardiol Rep. 2007; 9(3): 236-41. PMid:17470337 http://dx.doi.org/10.1007/BF02938356

[20] Nymo SH, Hulthe J, Ueland T, et al. Inflammatory cytokines in chronic heart failure: interleukin-8 is associated with adverse outcome. Results from CORONA. Eur J Heart Fail. 2014; 16(1): 68-75. PMid:23918775 http://dx.doi.org/10.1093/eurjhf/hft125

[21] Damas JK, Eiken HG, Oie E, et al. Myocardial expression of CC- and CXC-chemokines and their receptors in human end-stage heart failure. Cardiovasc Res. 2000; 47(4): 778-87. http://dx.doi.org/10.1016/S0008-6363(00)00142-5

[22] Cappuzzello C, Di Vito L, Melchionna R, et al. Increase of plasma IL-9 and decrease of plasma IL-5, IL-7, and IFN-gamma in patients with chronic heart failure. J Transl Med. 2011; 9: 28. PMid:21418620 http://dx.doi.org/10.1186/1479-5876-9-28

[23] Chan ED, Iseman MD. Underlying host risk factors for nontuberculous mycobacterial lung disease. Semin Respir Crit Care Med. 2013; 34(1): 110-23. PMid:23460011 http://dx.doi.org/10.1055/s-0033-1333573

[24] Chalermskulrat W, Gilbey JG, Donohue JF. Nontuberculous mycobacteria in women, young and old. Clin Chest Med. 2002; 23(3): 675-86. http://dx.doi.org/10.1016/S0272-5231(02)00010-2

[25] Griffith DE, Aksamit T, Brown-Elliott BA, et al. An official ATS/IDSA statement: diagnosis, treatment, and prevention of nontuberculous mycobacterial diseases. Am J Respir Crit Care Med. 2007; 175(4): 367-416.

[26] Rodvold KA. Clinical Pharmacokinetics of Clarithromycin. Clin Pharmacokinet. 1999; 37(5): 385-398.

[27] Wallace RJ, Brown BA, Griffith DE. Drug intolerance to high-dose clarithromycin among elderly patients. Diagn Microbiol Infect Dis. 1993; 16(3): 215-21. http://dx.doi.org/10.1016/0732-8893(93)90112-K

[28] Adjemian J, Prevots DR, Gallagher J, et al. Lack of adherence to evidence-based treatment guidelines for nontuberculous mycobacterial lung disease. Ann Am Thorac Soc. 2014; 11(1): 9-16. PMid:24236749 http://dx.doi.org/10.1513/AnnalsATS.201304-085OC

[29] Fox JC, Szyjkowski RS, Sanderson SO, et al. Progressive cholestatic liver disease associated with clarithromycin treatment. J Clin Pharmacol. 2002; 42(6): 676-80. PMid:12043957 http://dx.doi.org/10.1177/00970002042006011 
[30] Sousa C, Correia J, Santos J, et al. Cholestatic hepatitis probably induced by clarithromycin. Gastroenterol Clin Biol. 1997; 21(8-9): 632-3. PMid:9587506

[31] Brown BA, Wallace RJ, Griffith DE, et al. Clarithromycin-induced hepatotoxicity. Clin Infect Dis. 1995; $20(4)$ : $1073-4$. PMid:7795060 http://dx.doi.org/10.1093/clinids/20.4.1073

[32] Shaheen N, Grimm IS. Fulminant hepatic failure associated with clarithromycin. Am J Gastroenterol. 1996; 91(2): $394-5$. PMid:8607519

[33] Tietz A, Heim MH, Eriksson U, et al. Fulminant liver failure associated with clarithromycin. Ann Pharmacother. 2003; 37(1): 57-60. PMid:12503933 http://dx.doi.org/10.1345/aph.1C171

[34] Pessayre D, Tinel M, Larrey D, et al. Inactivation of cytochrome P-450 by a troleandomycin metabolite. Protective role of glutathione. J Pharmacol Exp Ther. 1983; 224(3): 685-91. PMid:6600790

[35] van Ingen J, Egelund EF, Levin A, et al. The pharmacokinetics and pharmacodynamics of pulmonary Mycobacterium avium complex disease treatment. Am J Respir Crit Care Med. 2012; 186(6): 559-65. PMid:22744719 http://dx.doi.org/10.1164/rccm.201204-0682OC

[36] Zuckerman JM, Qamar F, Bono BR. Review of macrolides (azithromycin, clarithromycin), ketolids (telithromycin) and glycylcyclines (tigecycline). Med Clin North Am. 2011; 95(4): 761-91. PMid:21679791 http://dx.doi.org/10.1016/j.mcna.2011.03.012

[37] Koh WJ, Jeong BH, Jeon K, et al. Therapeutic drug monitoring in the treatment of Mycobacterium avium complex lung disease. Am J Respir Crit Care Med. 2012; 186(8): 797-802. PMid:22878282 http://dx.doi.org/10.1164/rccm.201206-1088OC

[38] Pasipanodya JG, Gumbo T. Drug concentration monitoring in Mycobacterium avium lung disease: problems with methods and conclusions. Am J Respir Crit Care Med. 2013; 187(5): 554-5. PMid:23457368 http://dx.doi.org/10.1164/ajrccm.187.5.554

[39] Ambrose PG, Bhavnani SM, Rubino CM, et al. Pharmacokinetics-pharmacodynamics of antimicrobial therapy: it's not just for mice anymore. Clin Infect Dis. 2007; 44(1): 79-86. PMid:17143821 http://dx.doi.org/10.1086/510079

[40] Schmalstieg AM, Srivastava S, Belkaya S, et al. The antibiotic resistance arrow of time: efflux pump induction is a general first step in the evolution of mycobacterial drug resistance. Antimicrob Agents Chemother. 2012; 56(9): 4806-15. PMid:22751536 http://dx.doi.org/10.1128/AAC.05546-11

[41] Kobashi Y, Abe M, Mouri K, et al. Relationship between clinical efficacy for pulmonary MAC and drug-sensitivity test for isolated MAC in a recent 6-year period. J Infect Chemother. 2012; 18(4): 436-43. PMid:22205543 http://dx.doi.org/10.1007/s10156-011-0351-x

[42] Griffith DE, Brown BA, Cegielski P, et al. Early results (at 6 months) with intermittent clarithromycin-including regimens for lung disease due to Mycobacterium avium Complex. Clin Infect Dis. 2000; 30: 288-92. PMid:10671330 http://dx.doi.org/10.1086/313644

[43] Wallace RJ, Brown-Elliott BA, McNulty S, et al. Macrolide/azalide therapy for nodular/bronchiectatic Mycobacterium avium complex lung disease. Chest. 2014; 146(2): 276-282. PMid:24457542 http://dx.doi.org/10.1378/chest.13-2538 